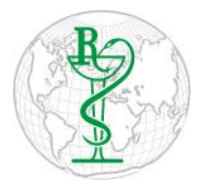

INDO GLOBAL JOURNAL OF

PHARMACEUTICAL SCIENCES

ISSN 2249- 1023

\title{
3,5-Diphenylpyrazolylethanone Derivatives as Selective Estrogen Receptor Modulators: Design, Synthesis, Biological Evaluation and Docking Analysis
}

\author{
Pritam N. Dube *, Vivekanand A. Chatpalliwar \\ Shree Neminath Jain Brahmacharyashram (Gurukul) Shriman Sureshdada Jain College of Pharmacy, Neminagar, Chandwad, Nashik-423101, \\ Maharashtra, India
}

Address for Correspondence: Pritam N. Dube, pritamdube@gmail.com

\begin{abstract}
Received:
06.01.2020

Accepted:

28.05.2020

Published:

18.08.2020

Keywords

Breast cancer,

Docking,

Pyrazole, SERM.
\end{abstract}

ABSTRACT: Novel 3, 5-pyrazolylethanone derivatives were synthesized and evaluated for their cytotoxic activity against estrogen receptor-positive MCF-7 and ZR-75-1 human breast cancer cell lines. Derivatives 4b and $4 \mathrm{c}$ were evaluated for in vivo anti-cancer activity by NMU induced mammary carcinoma in female Sprague-Dawley rats. The in-silico studies were conducted to attain a better insight into the interactions of these molecules with their target receptor to study their subtype selectivity and preferential binding site. The various orientations taken by ligands while binding the estrogen receptor- $\alpha$ were studied over 1ERR (PDB) using Schrodinger Maestro environments. () 2020 iGlobal Research and Publishing Foundation. All rights reserved.

Cite this article as: Dube, P.N.; Chatpalliwar, V.A. 3, 5-Diphenylpyrazolylethanone derivatives as selective estrogen receptor modulators: design, synthesis, biological evaluation and docking analysis. Indo Global J. Pharm. Sci., 2020; 10(1): 25-32. DOI: http://doi.org/10.35652/IGJPS.2020.10104 .

\section{INTRODUCTION}

Breast cancer $(\mathrm{BC})$ today is a leading cause of morbidity around the world, with the statistics showing increasing incidence rates.[1,2] Despite the available treatment choices available, up to this moment the fact remains that there is not a "cure" for BC or cancer in general. Therefore, medical scientists around the world are striving to come up with novel treatments that could enhance the "battle" against cancer.[2-4] The novel methods include hormonal therapy using selective estrogen receptor modulators like tamoxifen, raloxifene, etc. [2,5]

Several food components play an important role that inhibits the initiation and progression of cancer or otherwise in the prevention of disease govern by epidemiological and animal studies.[6-8] The presence of numerous chalcone analogs in dietary compounds has beneficial effects such as antioxidant and free radical scavenging properties.[9,10] Herein, we have designed and synthesized 3, 5-diphenylpyrazolethanone (chalcone analogs) as Selective Estrogen Receptor Modulators (Figure 1, Scheme 1). The synthesized derivatives were screen for their anticancer activity on human breast cancer cell lines and animal models.

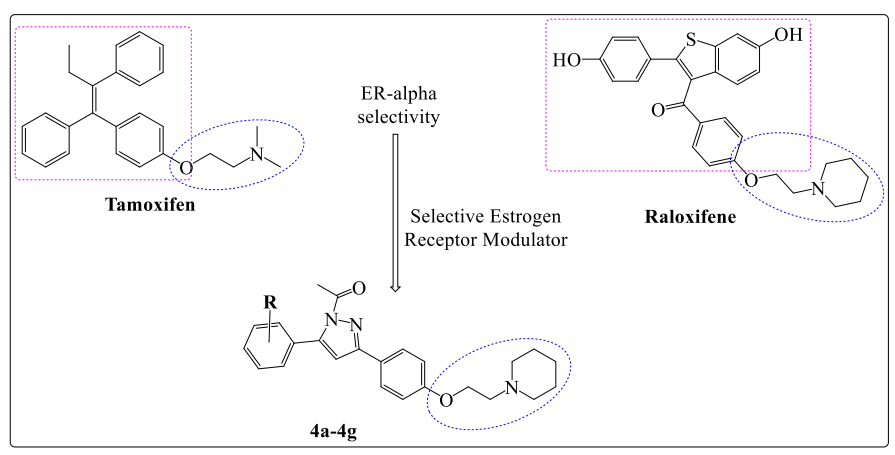

Figure 1: Designing of 3, 5-pyrazole Scaffold as Novel SERM 
Indo Global Journal of Pharmaceutical Sciences, 2020; 10(1): 25-32

\section{MATERIALS AND METHODS}

\section{Chemistry}

AR/LR grade reagents were used after purifying and drying appropriately. Characterization of the synthesized compounds is mentioned in the individual descriptions. Melting points were recorded in open capillaries with electrical melting point apparatus and were uncorrected. The yield of respective processes are mentioned in parentheses following the m. p. of respective derivatives. Silica gel $\mathrm{G}$ plates activated at $110{ }^{\circ} \mathrm{C}$ for $30 \mathrm{~min}$ were used for TLC, developed using the solvent system: (a) Chloroform: Ethyl acetate (3:7); (b) Benzene: Methanol (4:1); (c) Benzene: Methanol (9:1), and are enclosed in parentheses herein during individual characterization of the compound. The developed TLC plates were examined for colored spots that were apparent in exposing them to iodine vapors. The $\mathrm{R}_{f}$ values of purified compounds are represented herein as individual characterization. Single spot TLC, using various solvent systems, ascertained the purity of the compounds. Percentage yields shown are approximate along with the solvent used for crystallization in case of solid compounds and are mentioned within brackets after melting point (m. p.) reported in ${ }^{\circ} \mathrm{C}$. IR spectra of all synthesized compounds were recorded on JASCO FT-IR 4000 spectrophotometer using $\mathrm{KBr}$ as diluents, and are expressed herein $\mathrm{cm}^{-1}$. The ${ }^{1} \mathrm{H}$ NMR spectra were recorded on Bruker Avance (400 MHz) Spectrometer in DMSO solutions, whereas and ${ }^{13} \mathrm{C}$ NMR spectra were recorded with $100 \mathrm{MHz}$ on the same facility, using TMS as an internal reference. Chemical shifts $(\delta)$ in ppm are mentioned along with coupling frequencies as singlet $(s)$, doublet $(d)$, triplet $(t)$ and multiplet $(m)$; Mass spectra were recorded on a Varian Inc, 410 Prostar Binary $L C$ with 500 MS IT PDA Detectors.

\section{Synthesis of 1-(4-hydroxyphenyl)-5-phenylprop-2-en-1-one} [The Chalcones] (2a-2g)

A mixture of 4-hydroxyacetophenone $(3.1 \mathrm{mmol}, 700 \mathrm{mg})$, the appropriate commercially available appropriately substituted benzaldehyde a-g $(3.7 \mathrm{mmol})$ and $20 \%$ aq. $\mathrm{NaOH}(1 \mathrm{~mL})$ in $\mathrm{MeOH}(10 \mathrm{~mL})$ was stirred at room temperature. The reaction progress was monitored by TLC. After completion of the reaction, the reaction mixture was quenched with water and extracted with $\mathrm{CHCl}_{3}$. Then the organic phase was treated with anhydrous magnesium sulfate and the solvent was evaporated in vacuum. The dark viscous liquids either precipitate obtained was purified by column chromatography on silica gel employing 15:1 of $\mathrm{CHCl}_{3}-\mathrm{MeOH}$ as eluent. All the compounds were obtained as yellow to red viscous liquids.[11]

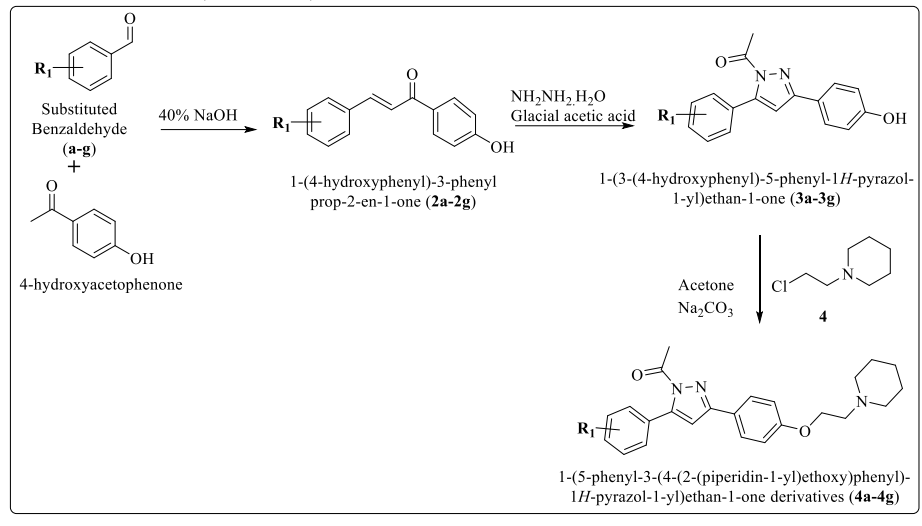

Scheme 1: Synthesis of designed compounds (4a-4g).

Synthesis of 1-(3-(4-hydroxyphenyl)-5-phenyl-4, 5dihydro-1H-pyrazol-1-yl-ethan-1-one (3a-3g)

A mixture of above mentioned chalcones $2 \mathbf{a}-\mathbf{g}(0.23 \mathrm{mmol})$, hydrazine hydrate $(0.5 \mathrm{mmol})$ and formic acid $(2 \mathrm{~mL})$ was subjected to reflux for 2-4 h with continuous stirring. The reaction progress was monitored by TLC. After completion of the reaction, ethanol was added and the precipitate formed was filtered. The filtrate was extracted using $\mathrm{CHCl}_{3}$, the organic phase was treated with anhydrous magnesium sulfate and the solvent was evaporated in vacuo. The viscous liquids obtained were washed with brine to form the compounds $\mathbf{3 a}-\mathbf{g}$.

Synthesis of 1-(5-phenyl-3-(4-(2-(piperidin-1-ylethoxy)phenyl)-1H-pyrazol-1-yl)ethan-1-one derivatives (4a-4g)

A mixture of compounds 3a-g (3 g), 1-(2chloroethyl)piperidine hydrochloride 4 (25.59 mmol, $1.8 \mathrm{~g})$, in acetic acid $(20 \mathrm{~mL})$, was subjected to refluxing with continuous stirring for $24 \mathrm{~h}$ in the presence of anhydrous potassium carbonate $(52 \mathrm{mmol}, 7.2 \mathrm{~g})$. The reaction progress was monitored by TLC. After completion of the reaction, the resultant suspension was cooled to room temperature, quenched with cold water and the crude compound was extracted with $\mathrm{CHCl}_{3}$. Then the organic phase was treated with anhydrous magnesium sulfate and the solvent was evaporated in vacuo. The obtained derivatives $\mathbf{4 a - 4 g}$, were purified by column chromatography on silica gel employing 30:1 $\mathrm{CHCl}_{3}-\mathrm{MeOH}$ as eluent.

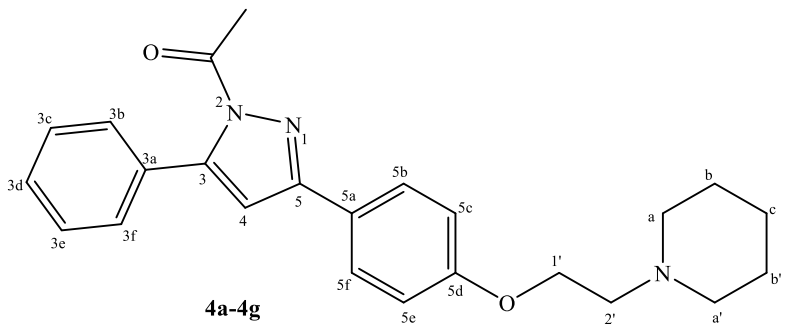

Figure 2: Structure and numerical assignment of synthesized derivatives 


\section{Indo Global Journal of Pharmaceutical Sciences, 2020; 10(1): 25-32}

\section{1-(5-phenyl-3-(4-(2-(piperidin-1-yl)ethoxy)phenyl)-1 H-} pyrazol-1-yl)ethan-1-one (4a)

MP: $144-146^{\circ} \mathrm{C}$ (82.28); $\mathrm{R}_{\mathrm{f}}$ : 0.72 (a); $\mathrm{MW}: 389.23$; MF: $\mathrm{C}_{24} \mathrm{H}_{27} \mathrm{~N}_{3} \mathrm{O}_{2}$; IR (KBr): 695 (Ar-H), $1186(\mathrm{C}-\mathrm{O}), 1326(\mathrm{C}-\mathrm{N})$, $1534(\mathrm{C}=\mathrm{C}), 1719(\mathrm{C}=\mathrm{O}) \mathrm{cm}^{-1} ;{ }^{1} \mathrm{H}$ NMR (DMSO, 400MHz): $\delta=7.7-7.4(m, 5 \mathrm{H}, \mathrm{Ar}-\mathrm{H}), 7.1\left(m, 2 \mathrm{H}, \mathrm{Ar}-\mathrm{H}_{5 \mathrm{~b}}, \mathrm{H}_{5 \mathrm{f}}\right), 6.9(m$, $\left.2 \mathrm{H}, \mathrm{Ar}-\mathrm{H}_{5 \mathrm{c}}, \mathrm{H}_{5 \mathrm{e}}\right), 4.1\left(t, 2 \mathrm{H}, \mathrm{CH}_{2}\right.$ at 1 '), $3.7(s, 1 \mathrm{H}, \mathrm{CH}$ of pyrazole), $2.8\left(t, 2 \mathrm{H}, \mathrm{CH}_{2}\right.$ at $\left.2^{\prime}\right), 2.4\left(m, 4 \mathrm{H}\right.$, piperidine $\mathrm{H}_{\mathrm{a}}$ \& $\left.\mathrm{H}_{\mathrm{a}^{\prime}}\right), 1.9\left(s, 3 \mathrm{H}, \mathrm{CH}_{3}-\mathrm{C}=\mathrm{O}\right), 1.4-1.3\left(m, 6 \mathrm{H}\right.$, piperidine $\mathrm{H}_{\mathrm{b}}, \mathrm{H}_{\mathrm{b}}$ $\left.\& \mathrm{H}_{\mathrm{c}}\right) ;{ }^{13} \mathrm{C}$ NMR (DMSO, 100MHz): $\delta=168.7(\mathrm{C}=\mathrm{O}), 157.4$ $\left(\mathrm{C}_{5 \mathrm{~d}}\right), 151.8\left(\mathrm{C}_{3}\right), 136.6\left(\mathrm{C}_{3 \mathrm{a}}\right), 134.1\left(\mathrm{C}_{5 \mathrm{a}}\right), 131.2\left(\mathrm{C}_{3 \mathrm{~d}}\right), 128.9$ $\left(\mathrm{C}_{3 \mathrm{c}}, \mathrm{C}_{3 \mathrm{e}}\right), 128.3\left(\mathrm{C}_{3 \mathrm{~b}}, \mathrm{C}_{3 \mathrm{f}}\right), 126.3\left(\mathrm{C}_{5 \mathrm{~b}}, \mathrm{C}_{5 \mathrm{f}}\right), 114.5\left(\mathrm{C}_{5 \mathrm{c}}, \mathrm{C}_{5 \mathrm{e}}\right)$, $66.4\left(\mathrm{C}_{1}\right), 65.8\left(\mathrm{C}_{5}\right), 60.6\left(\mathrm{C}_{2}\right), 57.2\left(\mathrm{C}_{\mathrm{a}}, \mathrm{C}_{\mathrm{a}^{\prime}}\right), 39.8\left(\mathrm{C}_{4}\right), 25.9$ $\left(\mathrm{C}_{\mathrm{b}}, \mathrm{C}_{\mathrm{b}}\right), 24.5\left(\mathrm{C}_{\mathrm{c}}\right), 23.2\left(\mathrm{CH}_{3}-\mathrm{C}=\mathrm{O}\right) ; \mathrm{MS}: \mathrm{m} / \mathrm{z}=389.23\left[\mathrm{M}^{+}\right.$, $100 \%], 390.23$ [M+1, 26\%], 390.22 [M+1, 1.1\%].

\section{1-[5-(4-chlorophenyl)-3-[4-(2-(piperidin-1-}

yl)ethoxy)phenyl]-1H-pyrazol-1-yl)ethan-1-one (4b)

MP: $182-184^{\circ} \mathrm{C}$ (72.84); $\mathrm{R}_{\mathrm{f}}$ : 0.74 (b); MW: 423.19; MF: $\mathrm{C}_{24} \mathrm{H}_{26} \mathrm{ClN}_{3} \mathrm{O}_{2}$; IR (KBr): 698 (Ar-H), 715 (C-Cl), 1190 (C-O), $1318(\mathrm{C}-\mathrm{N}), 1542(\mathrm{C}=\mathrm{C}), 1724(\mathrm{C}=\mathrm{O}) \mathrm{cm}^{-1}$; ${ }^{1} \mathrm{H}$ NMR (DMSO, $400 \mathrm{MHz}): \delta=7.7-7.3(m, 4 \mathrm{H}, \mathrm{Ar}-\mathrm{H}), 7.1\left(m, 2 \mathrm{H}, \mathrm{Ar}-\mathrm{H}_{5 \mathrm{~b}}, \mathrm{H}_{5 \mathrm{f}}\right)$, $6.8\left(m, 2 \mathrm{H}, \mathrm{Ar}-\mathrm{H}_{5 \mathrm{c}}, \mathrm{H}_{5 \mathrm{e}}\right), 4.2\left(t, 2 \mathrm{H}, \mathrm{CH}_{2}\right.$ at $\left.1^{\prime}\right), 3.7(s, 1 \mathrm{H}, \mathrm{CH}$ of pyrazole), $2.9\left(t, 2 \mathrm{H}, \mathrm{CH}_{2}\right.$ at 2 '), $2.4\left(m, 4 \mathrm{H}\right.$, piperidine $\mathrm{H}_{\mathrm{a}}$ \& $\left.\mathrm{H}_{\mathrm{a}}\right), 1.8\left(s, 3 \mathrm{H}, \mathrm{CH}_{3}-\mathrm{C}=\mathrm{O}\right), 1.4-1.3\left(m, 6 \mathrm{H}\right.$, piperidine $\mathrm{H}_{\mathrm{b}}$, $\left.\mathrm{H}_{\mathrm{b}}, \& \mathrm{H}_{\mathrm{c}}\right) ;{ }^{13} \mathrm{C}$ NMR (DMSO, 100MHz): $\delta=166.5(\mathrm{C}=\mathrm{O})$, $156.7\left(\mathrm{C}_{5 \mathrm{~d}}\right), 151.4\left(\mathrm{C}_{3}\right), 137.5\left(\mathrm{C}_{3 \mathrm{~d}}-\mathrm{Cl}\right), 136.4\left(\mathrm{C}_{3 \mathrm{a}}\right), 134.2$ $\left(\mathrm{C}_{5 \mathrm{a}}\right), 128.8\left(\mathrm{C}_{3 \mathrm{c}}, \mathrm{C}_{3 \mathrm{e}}\right), 128.2\left(\mathrm{C}_{3 \mathrm{~b}}, \mathrm{C}_{3 \mathrm{f}}\right), 126.2\left(\mathrm{C}_{5 \mathrm{~b}}, \mathrm{C}_{5 \mathrm{f}}\right), 112.4$ $\left(\mathrm{C}_{5 \mathrm{c}}, \mathrm{C}_{5 \mathrm{e}}\right), 67.2\left(\mathrm{C}_{1^{\prime}}\right), 65.6\left(\mathrm{C}_{5}\right), 60.1\left(\mathrm{C}_{2^{\prime}}\right), 56.5\left(\mathrm{C}_{\mathrm{a}}, \mathrm{C}_{\mathrm{a}^{\prime}}\right), 39.7$ $\left(\mathrm{C}_{4}\right), 26.0\left(\mathrm{C}_{\mathrm{b}}, \mathrm{C}_{\mathrm{b}}\right), 25.1\left(\mathrm{C}_{\mathrm{c}}\right), 23.3\left(\mathrm{CH}_{3}-\mathrm{C}=\mathrm{O}\right)$; MS: $\mathrm{m} / \mathrm{z}=$ $423.19\left[\mathrm{M}^{+}, 100 \%\right], 425.19$ [M+2, 2.7\%], 427.19 [M+4, 1\%].

\section{1-[5-(4-methoxyphenyl)-3-(4-(2-(piperidin-1-}

yl)ethoxy)phenyl]-1H-pyrazol-1-yl)ethan-1-one (4c)

MP: $164-166^{\circ} \mathrm{C}$ (63.94); $\mathrm{R}_{\mathrm{f}}$ : 0.68 (a); MW: 419.24; MF: $\mathrm{C}_{25} \mathrm{H}_{29} \mathrm{~N}_{3} \mathrm{O}_{3}$; IR (KBr): $715(\mathrm{Ar}-\mathrm{H}), 1182(\mathrm{C}-\mathrm{O}), 1322(\mathrm{C}-\mathrm{N})$, $1536(\mathrm{C}=\mathrm{C}), 1718(\mathrm{C}=\mathrm{O}) \mathrm{cm}^{-1} ;{ }^{1} \mathrm{H}$ NMR (DMSO, 400MHz): $\delta=7.8-7.4(m, 4 \mathrm{H}, \operatorname{Ar}-\mathrm{H}), 7.2\left(m, 2 \mathrm{H}, \operatorname{Ar}-\mathrm{H}_{5 \mathrm{~b}}, \mathrm{H}_{5 \mathrm{f}}\right), 6.7(m$, $\left.2 \mathrm{H}, \mathrm{Ar}-\mathrm{H}_{5 \mathrm{c}}, \mathrm{H}_{5 \mathrm{e}}\right), 4.2\left(t, 2 \mathrm{H}, \mathrm{CH}_{2}\right.$ at $\left.1^{\prime}\right), 3.9\left(s, 3 \mathrm{H}, \mathrm{OCH}_{3}\right), 3.6$ $\left(s, 1 \mathrm{H}, \mathrm{CH}\right.$ of pyrazole), $2.9\left(t, 2 \mathrm{H}, \mathrm{CH}_{2}\right.$ at 2 '), $2.3(m, 4 \mathrm{H}$, piperidine $\left.\mathrm{H}_{\mathrm{a}} \& \mathrm{H}_{\mathrm{a}}\right), 1.9\left(\mathrm{~s}, 3 \mathrm{H}, \mathrm{CH}_{3}-\mathrm{C}=\mathrm{O}\right), 1.4-1.2(m, 6 \mathrm{H}$, piperidine $\mathrm{H}_{\mathrm{b}}, \mathrm{H}_{\mathrm{b}}, \& \mathrm{H}_{\mathrm{c}}$ ); ${ }^{13} \mathrm{C}$ NMR (DMSO, $100 \mathrm{MHz}$ ): $\delta=$ $167.8(\mathrm{C}=\mathrm{O}), 162.4\left(\mathrm{C}_{3 \mathrm{~d}}-\mathrm{OCH}_{3}\right), 158.2\left(\mathrm{C}_{5 \mathrm{~d}}\right), 152.6\left(\mathrm{C}_{3}\right)$, $136.3\left(\mathrm{C}_{3 \mathrm{a}}\right), 133.9\left(\mathrm{C}_{5 \mathrm{a}}\right), 128.5\left(\mathrm{C}_{3 \mathrm{c}}, \mathrm{C}_{3 \mathrm{e}}\right), 127.4\left(\mathrm{C}_{3 \mathrm{~b}}, \mathrm{C}_{3 \mathrm{f}}\right)$, $126.4\left(\mathrm{C}_{5 \mathrm{~b}}, \mathrm{C}_{5 \mathrm{f}}\right), 113.7\left(\mathrm{C}_{5 \mathrm{c}}, \mathrm{C}_{5 \mathrm{e}}\right), 67.6\left(\mathrm{C}_{1}\right), 65.1\left(\mathrm{C}_{5}\right), 60.2$ $\left(\mathrm{C}_{2}{ }^{\prime}\right), 56.6\left(\mathrm{C}_{\mathrm{a}}, \mathrm{C}_{\mathrm{a}^{\prime}}\right), 54.6\left(\mathrm{OCH}_{3}\right), 39.8\left(\mathrm{C}_{4}\right), 26.2\left(\mathrm{C}_{\mathrm{b}}, \mathrm{C}_{\mathrm{b}^{\prime}}\right)$, $25.2\left(\mathrm{C}_{\mathrm{c}}\right), 23.4\left(\mathrm{CH}_{3}-\mathrm{C}=\mathrm{O}\right) ; \mathrm{MS}: \mathrm{m} / z=419.24\left[\mathrm{M}^{+}, 100 \%\right]$, $420.24[\mathrm{M}+1,27 \%]$.
1-(5-(2,

6-dichlorophenyl)-3-(4-(2-(piperidin-1yl)ethoxy)phenyl)-1 $\mathrm{H}$-pyrazol-1-yl)ethan-1-one (4d)

MP: $174-176^{\circ} \mathrm{C}$ (88.12); $\mathrm{R}_{\mathrm{f}}$ : 0.58 (c); MW: 457.15; MF: $\mathrm{C}_{24} \mathrm{H}_{25} \mathrm{Cl}_{2} \mathrm{~N}_{3} \mathrm{O}_{2}$; IR (KBr): 690 (Ar-H), 716 (C-Cl), 1195 (CO), $1327(\mathrm{C}-\mathrm{N}), 1540(\mathrm{C}=\mathrm{C}), 1722(\mathrm{C}=\mathrm{O}) \mathrm{cm}^{-1} ;{ }^{1} \mathrm{H}$ NMR (DMSO, 400MHz): $\delta=7.6-7.3(m, 3 \mathrm{H}, \mathrm{Ar}-\mathrm{H}), 7.0(\mathrm{~m}, 2 \mathrm{H}, \mathrm{Ar}-$ $\left.\mathrm{H}_{5 \mathrm{~b}}, \mathrm{H}_{5 \mathrm{f}}\right), 6.7\left(m, 2 \mathrm{H}, \mathrm{Ar}-\mathrm{H}_{5 \mathrm{c}}, \mathrm{H}_{5 \mathrm{e}}\right), 4.2\left(t, 2 \mathrm{H}, \mathrm{CH}_{2}\right.$ at 1'), 3.7 $\left(s, 1 \mathrm{H}, \mathrm{CH}\right.$ of pyrazole), $2.9\left(t, 2 \mathrm{H}, \mathrm{CH}_{2}\right.$ at $\left.2^{\prime}\right), 2.4(\mathrm{~m}, 4 \mathrm{H}$, piperidine $\left.\mathrm{H}_{\mathrm{a}} \& \mathrm{H}_{\mathrm{a}^{\prime}}\right), 1.8\left(\mathrm{~s}, 3 \mathrm{H}, \mathrm{CH}_{3}-\mathrm{C}=\mathrm{O}\right), 1.4-1.3(\mathrm{~m}, 6 \mathrm{H}$, piperidine $\mathrm{H}_{\mathrm{b}}, \mathrm{H}_{\mathrm{b}}, \& \mathrm{H}_{\mathrm{c}}$ ); ${ }^{13} \mathrm{C}$ NMR (DMSO, $100 \mathrm{MHz}$ ): $\delta=$ $166.8(\mathrm{C}=\mathrm{O}), 156.4\left(\mathrm{C}_{5 \mathrm{~d}}\right), 151.2\left(\mathrm{C}_{3}\right), 138.1\left(\mathrm{C}_{3 \mathrm{a}}\right), 135.4$ $\left(\mathrm{C}_{3 \mathrm{~b}, 3 \mathrm{f}}-\mathrm{Cl}\right), 134.2\left(\mathrm{C}_{5 \mathrm{a}}\right), 130.6\left(\mathrm{C}_{3 \mathrm{~d}}\right), 129.1\left(\mathrm{C}_{3 \mathrm{c}}, \mathrm{C}_{3 \mathrm{e}}\right), 126.5$ $\left(\mathrm{C}_{5 \mathrm{~b}}, \mathrm{C}_{5 \mathrm{f}}\right), 112.7\left(\mathrm{C}_{5 \mathrm{c}}, \mathrm{C}_{5 \mathrm{e}}\right), 67.3\left(\mathrm{C}_{1^{\prime}}\right), 65.8\left(\mathrm{C}_{5}\right), 60.2\left(\mathrm{C}_{2^{\prime}}\right)$, $57.1\left(\mathrm{C}_{\mathrm{a}}, \mathrm{C}_{\mathrm{a}^{\prime}}\right), 39.3\left(\mathrm{C}_{4}\right), 25.8\left(\mathrm{C}_{\mathrm{b}}, \mathrm{C}_{\mathrm{b}^{\prime}}\right), 25.0\left(\mathrm{C}_{\mathrm{c}}\right), 23.4\left(\mathrm{CH}_{3-}\right.$ $\mathrm{C}=\mathrm{O})$; $\mathrm{MS}: \mathrm{m} / \mathrm{z}=457.15\left[\mathrm{M}^{+}, 100 \%\right], 459.15[\mathrm{M}+2,63.9 \%]$, $461.15[\mathrm{M}+4,1.7 \%]$.

1-(5-(2, 4-dimethoxyphenyl)-3-(4-(2-(piperidin-1yl)ethoxy)phenyl)-1H-pyrazol-1-yl)ethan-1-one (4e)

MP: $182-184^{\circ} \mathrm{C}$ (80.42); $\mathrm{R}_{\mathrm{f}}$ : 0.56 (a); MW: 449.25; MF: $\mathrm{C}_{26} \mathrm{H}_{31} \mathrm{~N}_{3} \mathrm{O}_{4}$; IR (KBr): 721 (Ar-H), 1178 (C-O), $1326(\mathrm{C}-\mathrm{N})$, $1534(\mathrm{C}=\mathrm{C}), 1714(\mathrm{C}=\mathrm{O}) \mathrm{cm}^{-1}$; ${ }^{1} \mathrm{H}$ NMR (DMSO, 400MHz): $\delta=7.8-7.5(m, 3 \mathrm{H}, \mathrm{Ar}-\mathrm{H}), 7.2\left(m, 2 \mathrm{H}, \mathrm{Ar}-\mathrm{H}_{5 \mathrm{~b}}, \mathrm{H}_{5 \mathrm{f}}\right), 6.8(m$, $\left.2 \mathrm{H}, \mathrm{Ar}-\mathrm{H}_{5 \mathrm{c}}, \mathrm{H}_{5 \mathrm{e}}\right), 4.2\left(t, 2 \mathrm{H}, \mathrm{CH}_{2}\right.$ at $\left.1^{\prime}\right), 3.9\left(s, 6 \mathrm{H}, \mathrm{OCH}_{3}\right), 3.6$ $(s, 1 \mathrm{H}, \mathrm{CH}$ of pyrazole $), 3.0\left(t, 2 \mathrm{H}, \mathrm{CH}_{2}\right.$ at $\left.2 '\right), 2.2(m, 4 \mathrm{H}$, piperidine $\left.\mathrm{H}_{\mathrm{a}} \& \mathrm{H}_{\mathrm{a}^{\prime}}\right), 1.9\left(\mathrm{~s}, 3 \mathrm{H}, \mathrm{CH}_{3}-\mathrm{C}=\mathrm{O}\right), 1.4-1.2(\mathrm{~m}, 6 \mathrm{H}$, piperidine $\mathrm{H}_{\mathrm{b}}, \mathrm{H}_{\mathrm{b}}, \& \mathrm{H}_{\mathrm{c}}$ ); ${ }^{13} \mathrm{C}$ NMR (DMSO, $100 \mathrm{MHz}$ ): $\delta=$ $168.0(\mathrm{C}=\mathrm{O}), 163.7\left(\mathrm{C}_{3 \mathrm{~d}}-\mathrm{OCH}_{3}\right), 162.4\left(\mathrm{C}_{3 \mathrm{~b}}-\mathrm{OCH}_{3}\right), 157.9$ $\left(\mathrm{C}_{5 \mathrm{~d}}\right), 152.5\left(\mathrm{C}_{3}\right), 137.1\left(\mathrm{C}_{3 \mathrm{a}}\right), 133.8\left(\mathrm{C}_{5 \mathrm{a}}\right), 129.1\left(\mathrm{C}_{3 \mathrm{c}}, \mathrm{C}_{3 \mathrm{e}}\right)$, $127.6\left(\mathrm{C}_{3 \mathrm{f}}\right), 126.3\left(\mathrm{C}_{5 \mathrm{~b}}, \mathrm{C}_{5 \mathrm{f}}\right), 113.5\left(\mathrm{C}_{5 \mathrm{c}}, \mathrm{C}_{5 \mathrm{e}}\right), 67.5\left(\mathrm{C}_{1}\right), 65.2$ $\left(\mathrm{C}_{5}\right), 59.5\left(\mathrm{C}_{2}\right), 56.7\left(\mathrm{C}_{\mathrm{a}}, \mathrm{C}_{\mathrm{a}^{\prime}}\right), 53.4\left(\mathrm{OCH}_{3}\right), 39.2\left(\mathrm{C}_{4}\right), 26.1$ $\left(\mathrm{C}_{\mathrm{b}}, \mathrm{C}_{\mathrm{b}}\right), 25.1\left(\mathrm{C}_{\mathrm{c}}\right), 22.8\left(\mathrm{CH}_{3}-\mathrm{C}=\mathrm{O}\right)$; MS: $\mathrm{m} / \mathrm{z}=449.25\left[\mathrm{M}^{+}\right.$, $100 \%], 450.25[\mathrm{M}+1,28.1 \%]$.

1-(3-(4-(2-(piperidin-1-yl)ethoxy)phenyl)-5-(2, 3, 4trimethoxyphenyl)-1 $\mathrm{H}$-pyrazol-1-yl)ethan-1-one (4f)

MP: $188-190^{\circ} \mathrm{C}$ (77.64); $\mathrm{R}_{\mathrm{f}}: 0.62$ (a); MW: 479.26; MF: $\mathrm{C}_{27} \mathrm{H}_{33} \mathrm{~N}_{3} \mathrm{O}_{5}$; IR (KBr): 733 (Ar-H), 1181 (C-O), 1325 (C-N), $1529(\mathrm{C}=\mathrm{C}), 1722(\mathrm{C}=\mathrm{O}) \mathrm{cm}^{-1} ;{ }^{1} \mathrm{H}$ NMR (DMSO, 400MHz): $\delta=7.8-7.4(m, 2 \mathrm{H}, \mathrm{Ar}-\mathrm{H}), 7.2\left(m, 2 \mathrm{H}, \mathrm{Ar}-\mathrm{H}_{5 \mathrm{~b}}, \mathrm{H}_{5 \mathrm{f}}\right), 6.7(m$, $\left.2 \mathrm{H}, \mathrm{Ar}-\mathrm{H}_{5 \mathrm{c}}, \mathrm{H}_{5 \mathrm{e}}\right), 4.2\left(t, 2 \mathrm{H}, \mathrm{CH}_{2}\right.$ at 1 '), $3.8\left(s, 9 \mathrm{H}, \mathrm{OCH}_{3}\right), 3.5$ $(s, 1 \mathrm{H}, \mathrm{CH}$ of pyrazole $), 2.8\left(t, 2 \mathrm{H}, \mathrm{CH}_{2}\right.$ at $\left.2 '\right), 2.1(m, 4 \mathrm{H}$, piperidine $\left.\mathrm{H}_{\mathrm{a}} \& \mathrm{H}_{\mathrm{a}}\right), 1.8\left(\mathrm{~s}, 3 \mathrm{H}, \mathrm{CH}_{3}-\mathrm{C}=\mathrm{O}\right), 1.5-1.3(\mathrm{~m}, 6 \mathrm{H}$, piperidine $\mathrm{H}_{\mathrm{b}}, \mathrm{H}_{\mathrm{b}}, \& \mathrm{H}_{\mathrm{c}}$ ); ${ }^{13} \mathrm{C}$ NMR (DMSO, $100 \mathrm{MHz}$ ): $\delta=$ $167.4(\mathrm{C}=\mathrm{O}), 161.5\left(\mathrm{C}_{3 \mathrm{~d}}-\mathrm{OCH}_{3}\right), 159.8\left(\mathrm{C}_{3 \mathrm{~b}}-\mathrm{OCH}_{3}\right), 158.0$ $\left(\mathrm{C}_{5 \mathrm{~d}}\right), 153.2\left(\mathrm{C}_{3}\right), 151.2\left(\mathrm{C}_{3 \mathrm{c}}-\mathrm{OCH}_{3}\right), 137.5\left(\mathrm{C}_{3 \mathrm{a}}\right), 133.7\left(\mathrm{C}_{5 \mathrm{a}}\right)$, $129.2\left(\mathrm{C}_{3 \mathrm{e}}\right), 127.8\left(\mathrm{C}_{3 \mathrm{f}}\right), 126.4\left(\mathrm{C}_{5 \mathrm{~b}}, \mathrm{C}_{5 \mathrm{f}}\right), 111.6\left(\mathrm{C}_{5 \mathrm{c}}, \mathrm{C}_{5 \mathrm{e}}\right), 67.3$ $\left(\mathrm{C}_{1}\right), 64.9\left(\mathrm{C}_{5}\right), 59.1\left(\mathrm{C}_{2}\right), 56.8\left(\mathrm{C}_{\mathrm{a}}, \mathrm{C}_{\mathrm{a}^{\prime}}\right), 54.4,53.5\left(\mathrm{OCH}_{3}\right)$, $38.1\left(\mathrm{C}_{4}\right), 26.3\left(\mathrm{C}_{\mathrm{b}}, \mathrm{C}_{\mathrm{b}}\right), 25.7\left(\mathrm{C}_{\mathrm{c}}\right), 22.5\left(\mathrm{CH}_{3}-\mathrm{C}=\mathrm{O}\right)$; MS: $\mathrm{m} / \mathrm{z}$ $=479.26\left[\mathrm{M}^{+}, 100 \%\right], 480.26[\mathrm{M}+1,29.2 \%]$. 


\section{Indo Global Journal of Pharmaceutical Sciences, 2020; 10(1): 25-32}

\section{1-(5-(4-fluorophenyl)-3-(4-(2-(piperidin-1-}

yl)ethoxy)phenyl)-1 $H$-pyrazol-1-yl)ethan-1-one $(4 \mathrm{~g})$

MP: $156-158^{\circ} \mathrm{C}(82.24)$; $\mathrm{R}_{\mathrm{f}}$ : 0.72 (b); MW: 407.22; MF:

$\mathrm{C}_{24} \mathrm{H}_{26} \mathrm{FN}_{3} \mathrm{O}_{2}$; IR (KBr): 699 (Ar-H), 718 (C-F), 1194 (C-O), $1308(\mathrm{C}-\mathrm{N}), 1528(\mathrm{C}=\mathrm{C}), 1725(\mathrm{C}=\mathrm{O}) \mathrm{cm}^{-1}$; ${ }_{1}^{1} \mathrm{H}$ NMR (DMSO, $400 \mathrm{MHz}): \delta=7.7-7.3(m, 4 \mathrm{H}, \mathrm{Ar}-\mathrm{H}), 7.1\left(m, 2 \mathrm{H}, \mathrm{Ar}-\mathrm{H}_{5 \mathrm{~b}}, \mathrm{H}_{5 \mathrm{f}}\right)$, $6.8\left(m, 2 \mathrm{H}, \mathrm{Ar}-\mathrm{H}_{5 \mathrm{c}}, \mathrm{H}_{5 \mathrm{e}}\right), 4.3\left(t, 2 \mathrm{H}, \mathrm{CH}_{2}\right.$ at 1 '), $3.7(s, 1 \mathrm{H}, \mathrm{CH}$ of pyrazole), $2.8\left(t, 2 \mathrm{H}, \mathrm{CH}_{2}\right.$ at 2 '), $2.4\left(m, 4 \mathrm{H}\right.$, piperidine $\mathrm{H}_{\mathrm{a}}$ \& $\left.\mathrm{H}_{\mathrm{a}}{ }^{\prime}\right), 1.8\left(\mathrm{~s}, 3 \mathrm{H}, \mathrm{CH}_{3}-\mathrm{C}=\mathrm{O}\right), 1.4-1.2\left(m, 6 \mathrm{H}\right.$, piperidine $\mathrm{H}_{\mathrm{b}}$, $\left.\mathrm{H}_{\mathrm{b}}, \& \mathrm{H}_{\mathrm{c}}\right) ;{ }^{13} \mathrm{C}$ NMR (DMSO, 100MHz): $\delta=164.1(\mathrm{C}=\mathrm{O})$, $156.8\left(\mathrm{C}_{5 \mathrm{~d}}\right), 152.7\left(\mathrm{C}_{3}\right), 136.8\left(\mathrm{C}_{3 \mathrm{~d}} \mathrm{-F}\right), 135.9\left(\mathrm{C}_{3 \mathrm{a}}\right), 133.7\left(\mathrm{C}_{5 \mathrm{a}}\right)$, $128.7\left(\mathrm{C}_{3 \mathrm{c}}, \mathrm{C}_{3 \mathrm{e}}\right), 128.3\left(\mathrm{C}_{3 \mathrm{~b}}, \mathrm{C}_{3 \mathrm{f}}\right), 126.4\left(\mathrm{C}_{5 \mathrm{~b}}, \mathrm{C}_{5 \mathrm{f}}\right), 113.5\left(\mathrm{C}_{5 \mathrm{c}}\right.$, $\left.\mathrm{C}_{5 \mathrm{e}}\right), 67.3\left(\mathrm{C}_{1^{\prime}}\right), 65.7\left(\mathrm{C}_{5}\right), 60.2\left(\mathrm{C}_{2^{\prime}}\right), 56.6\left(\mathrm{C}_{\mathrm{a}}, \mathrm{C}_{\mathrm{a}^{\prime}}\right), 38.4\left(\mathrm{C}_{4}\right)$, $26.1\left(\mathrm{C}_{\mathrm{b}}, \mathrm{C}_{\mathrm{b}}\right), 25.2\left(\mathrm{C}_{\mathrm{c}}\right), 23.8\left(\mathrm{CH}_{3}-\mathrm{C}=\mathrm{O}\right) ; \mathrm{MS}: \mathrm{m} / z=407.22$ $\left[\mathrm{M}^{+}, 100 \%\right], 409.22[\mathrm{M}+2,2.7 \%]$.

\section{Biological Evaluation}

\section{In vitro}

Anti-cancer tests were performed using SRB assay protocols [12], each drug is tested at 4 dose levels (1x10-7M, $1 \times 10-6 \mathrm{M}$, $1 \times 10-5 \mathrm{M}, 1 \times 10-4 \mathrm{M}$, or $10,20,40,80 \mu \mathrm{g} / \mathrm{mL})$. Appropriate positive controls are run in each experiment and each experiment is repeated thrice. Results are given in terms of GI50, TGI and LC50 values. The compounds were tested for their cytotoxic assay using MCF-7 and ZR-75-1 breast cancer cell lines.

\section{In vivo}

Animal experiments were approved by the IAEC of the concerned center. Female virgin SD rats were obtained from Wockhardt Pvt. Ltd. (Aurangabad, India) at 35 days of age, and were housed at 6 per cage, maintained at $25 \pm 2{ }^{\circ} \mathrm{C}$ under $12 \mathrm{~h}$ dark/light cycles with access to standard diet and water $a b$ libitum. Solutions of carcinogen, $N$-Nitroso- $N$-methylurea (NMU), were purchased from Sigma (USA), were prepared afresh immediately before administration to the animals, using $3 \%$ acetic acid, normal saline, and NMU to get carcinogenic solution $10 \mathrm{mg} / \mathrm{mL}$. Solutions equivalent to $50 \mathrm{mg} \mathrm{NMU} / \mathrm{Kg}$ weight of animals were administered i.p. on the 50th and 57th day of age. Animals were grouped to have six each, and were as follows: Group I was negative control receiving only saline; Group II was experimental positive control receiving the carcinogenic solution. Group III - Group VI receiving Tamoxifen, 4b, 4c, and vehicle Tween 80, respectively. Animals in respective groups received the drug and the 3,5diphenylpyrazole derivatives, $10 \mathrm{mg} / \mathrm{Kg}$ each in Tween 80 , orally after a two week induction period, once a day for six weeks. Animals in the intact control group and untreated NMU group were given vehicle (Tween 80) according to the experimental protocol. Regular weekly palpation was carried until the detection of mammary tumors under light ether anesthesia.

At the end of the experimental period, all the rats were sacrificed by cervical dislocation. All gross palpable mammary lesions were excised, fixed in $10 \%$ phosphatebuffered neutral formalin, embedded in paraffin. The observations were scored as follows: The time taken since the day of challenge for first incidence of tumor was noted as Tumor Latency Period (TLP), number of tumors/rat as marked as Tumor Burden. Size of every tumor was recorded using micrometer calliper, and volume was calculated using the formula $V=4 / 3 n r 3$, where, $r$ is half of the average diameter. Intermittently, blood samples were collected from those animals in the fifth week since NMU challenge, i.e. at the start of the treatment period, and in the tenth week from the challenge, i.e. at the end of treatment period, from retro orbital plexus in blood collection tubes and were analyzed for estrogen level measurement. These were analyzed for estrogen levels by ELISA.[13]

\section{Molecular Docking studies}

Simulations were performed using solutions provided by Schrödinger. [14] The aforementioned structures were constructed using ACD Freeware and converted into *.mol files, before transferring into $O P L S$. Three dimensional crystal structure (PDB: 1ERR, Sequence identity $95 \%$ ) was obtained and a cavity grid was prepared using GLIDE. The three dimensional structures in flexible modes (generated using LigPrep) were docked in the rigid grid of the protein. The study was carried with and without bound-water molecules within $5 \AA$ Á vicinity of the cavity, whereas, hydrogen-bonds, ring interactions, $\pi-\pi$ stacking opted for the study. These studies were carried using the Xtra Presicion (XP) module of the Schrödinger software for scoring the energy minimum.

\section{RESULTS AND DISCUSSION}

\section{Chemistry}

The condensation reaction of Claisen-Schmidt between the 4hydroxyacetophenone and different benzaldehydes a-g, was carried out affording the corresponding chalcones 2a-g (Scheme 1). [11] In second step, 1, 3-dielectrophile cyclization reactions of chalcones $\mathbf{2 a - g}$ were carried out, employing a three-part methodology, in which the chalcones were mixed with hydrazine hydrate and acetic acid. Lastly, the designed compounds (4a-4g) were synthesized by alkylation of the hydroxyl group (3a-g) with the 1-(2chloroethyl)piperidine Hydrochloride (4) and $\mathrm{K}_{2} \mathrm{CO}_{3}$. DMF was used as the solvent of the reaction, which was carried out 
Indo Global Journal of Pharmaceutical Sciences, 2020; 10(1): 25-32

under reflux. The designed compounds were characterized by using FT-IR, ${ }^{1} \mathrm{H}-\mathrm{NMR},{ }^{13} \mathrm{C}-\mathrm{NMR}$ and LC-MS techniques that allowed elucidating its structures.

\section{Biological Evaluation}

The synthesized compounds were evaluated for their in vitro anticancer activity against ER $\alpha+$ human breast carcinoma cell line (MCF-7 \& Zr-75-1) using the SRB assay. [12] Cytotoxicity results of synthesized compounds with their $\mathrm{LC}_{50}$, TGI and $\mathrm{GI}_{50}$ value on breast cancer cell line is enlisted in Table 1. The concentration of candidate derivatives in $\mu \mathrm{g} / \mathrm{mL}$ inhibiting $50 \%$ of cancerous cell growth is expressed as $\mathrm{GI}_{50}$, the concentration inhibiting total cancerous cell growth expressed as TGI, and the concentration inducing cytocidal effect in $50 \%$ population of cancerous cell expressed as $\mathrm{LC}_{50}$. The percentages of inhibition/cytocidal effects induced by them are calculated considering the effect of standard Tamoxifen as 100.

The derivatives $\mathbf{4 b}$ and $\mathbf{4 c}$ show the most potent activity amongst the lot. The compound $\mathbf{4 b}$ is 4-chloro substituted whereas $\mathbf{4 c}$ compound contains 4-methoxy substitution. The di-halo substituted and trimethoxy substituted compounds shows very less cytocidal activity.

Table 1: In-vitro anticancer activity $(\mu \mathrm{g} / \mathrm{mL})$ of synthesized compounds

\begin{tabular}{|c|c|c|c|c|c|c|c|}
\hline \multirow{2}{*}{ Sr. No. } & \multirow{2}{*}{$\mathbf{R}$} & \multicolumn{3}{|c|}{ MCF-7 } & \multicolumn{3}{|c|}{ ZR-75-1 } \\
\hline & & $\mathbf{L C}_{50}{ }^{a}$ & TGI $^{b}$ & GI $_{50}{ }^{c}$ & $\mathrm{LC}_{50}$ & TGI & GI $_{50}$ \\
\hline $4 a$ & & 88.3 & 61.7 & 22.9 & $>100$ & $>100$ & 36.7 \\
\hline $4 b$ & & 81.3 & 38.4 & 12.2 & $>100$ & $>100$ & 19.8 \\
\hline $4 c$ & & 54.7 & 39.2 & 10.8 & $>100$ & $>100$ & 16.4 \\
\hline $4 d$ & & 62.6 & 41.4 & 16.2 & $>100$ & $>100$ & 32.8 \\
\hline $4 e$ & & 70.8 & 78.9 & 24.8 & $>100$ & $>100$ & 58.4 \\
\hline $4 f$ & & 94.8 & 79.2 & 39.9 & $>100$ & $>100$ & 57.9 \\
\hline $4 g$ & & 91.4 & 89.9 & 43.5 & $>100$ & $>100$ & 82.6 \\
\hline TAM & - & 39.5 & 16.3 & $<10$ & $>100$ & $>100$ & $<0.1$ \\
\hline
\end{tabular}

Most potent compounds shown by bold text as compare to standard

TAM tamoxifen

${ }^{a}$ Compound concentration that produces $50 \%$ cytocidal effect

${ }^{\mathrm{b}}$ Compound concentration that produces total growth inhibition

${ }^{\mathrm{c}}$ Compound concentration that produces $50 \%$ growth inhibition 
Indo Global Journal of Pharmaceutical Sciences, 2020; 10(1): 25-32

Oral administration of $\mathbf{4 b}$ and $\mathbf{4 c}$ to the laboratory animals induced with mammary carcinogenesis resulted in decreased incidences of the tumor, reduced tumor burden and tumor volume. The recorded observations are tabulated in Table 2. The data from tumor incidences were statistically analyzed by the Chi-Square test for the assessment of significance in protection against tumor occurrence. A difference of $P<0.05$ was considered significant in all cases. The Chi-square analysis indicated that drug/tamoxifen treatment exhibited significant anti-tumor activity $[(\mathrm{d} f=13.37,5), P<0.0201]$ against NMU ( $N$-Nitroso- $N$-methylurea)-induced breast tumor as observed by reduction in the tumor incidences. Corresponding to this, there was a reduction in the tumor volume $\left(\mathrm{mm}^{3}\right)$ in both groups.

Estrogen level of all groups measured on day 35 of intoxication (90th day since birth) was compared with the control and NMU group. [13] Mean observed estrogen levels given in Table $\mathbf{3}$. The results show that the compound $\mathbf{4 b}$ and $\mathbf{4 c}$ had protuberant affinity towards estrogen receptor, similar to standard Tamoxifen (TAM).

\section{Docking Study}

Computer-aided simulations assisted in selecting molecules from the designed set. The results indicated acceptable reliability of the parameters specified in Maestro- Glide in reproducing the binding mode for these compounds. After the successful reproduction of the binding mode of Raloxifen (RAL) (Fig. 3a), the docking method was used to search for the binding modes of the whole data set. Binding propensity was analyzed by docking on 1ERR, the crystal structure of ER $\alpha .[14]$ All the compounds were successfully docked into the binding pocket of ER $\alpha$. In this research, the attention has been focused on protein- ligand interactions of the unsubstituted compound $\mathbf{4 a}$, the p-chloro substituted compound $\mathbf{4 b}$ and the p-fluro-substituted compound $\mathbf{4 g}$ to illustrate the interaction mechanism.

Table 2: Effect of treatment on mammary tumorigenesis

\begin{tabular}{ccccccc}
\hline Group & Treatment & $\begin{array}{c}\text { No. of rats with } \\
\text { tumor }\end{array}$ & Incidences (\%) & $\begin{array}{c}\text { Tumor } \\
\text { Latency } \\
(\text { week })\end{array}$ & Tumor burden & $\begin{array}{c}\text { Tumor volume } \\
\left(\mathbf{m m}^{3}\right)\end{array}$ \\
\hline I & MNU & $6 / 6$ & 100 & $4.1 \pm 0.8$ & $3.1 \pm 0.14$ & $4.8 \pm 0.16$ \\
II & TAM & $2 / 6$ & 33.3 & $6.8 \pm 0.3$ & $6.2 \pm 0.21$ & $2.7 \pm 0.11$ \\
III & $\mathbf{4 b}$ & $2 / 6$ & 33.3 & $6.2 \pm 0.7$ & $6.3 \pm 0.03$ & $2.6 \pm 0.10$ \\
IV & $\mathbf{4 c}$ & $3 / 6$ & 50 & $6.1 \pm 0.6$ & $6.1 \pm 0.12$ & $3.1 \pm 0.5$ \\
\hline
\end{tabular}

Table 3: Estrogen levels (pg/mL) of animals on day 90

\begin{tabular}{ccc}
\hline Group & Treatment & $\begin{array}{c}\text { Estrogen level on 90 } \\
(\text { mean } \pm \text { SE })\end{array}$ \\
\hline I & Control & $17.1 \pm 1.14^{\mathrm{b}}$ \\
II & MNU & $34.25 \pm 2.1^{\mathrm{a}}$ \\
III & TAM & $18.13 \pm 1.01^{\mathrm{a}}$ \\
IV & $\mathbf{4 b}$ & $16.48 \pm 1.08^{\mathrm{a}}$ \\
V & $\mathbf{4 c}$ & $20.14 \pm 1.20^{\mathrm{a}}$ \\
\hline
\end{tabular}

The results are expressed as Mean \pm SEM. The data is analyzed using One-way Analysis of Variance (ANOVA) followed by Tukey's test. $(\mathrm{n}=6),{ }^{\mathrm{a}} \mathrm{P}<0.001,{ }^{\mathrm{b}} \mathrm{P}<0.01,{ }^{\mathrm{c}} \mathrm{P}<0.05,{ }^{\mathrm{d}}$ non significant. 
Indo Global Journal of Pharmaceutical Sciences, 2020; 10(1): 25-32

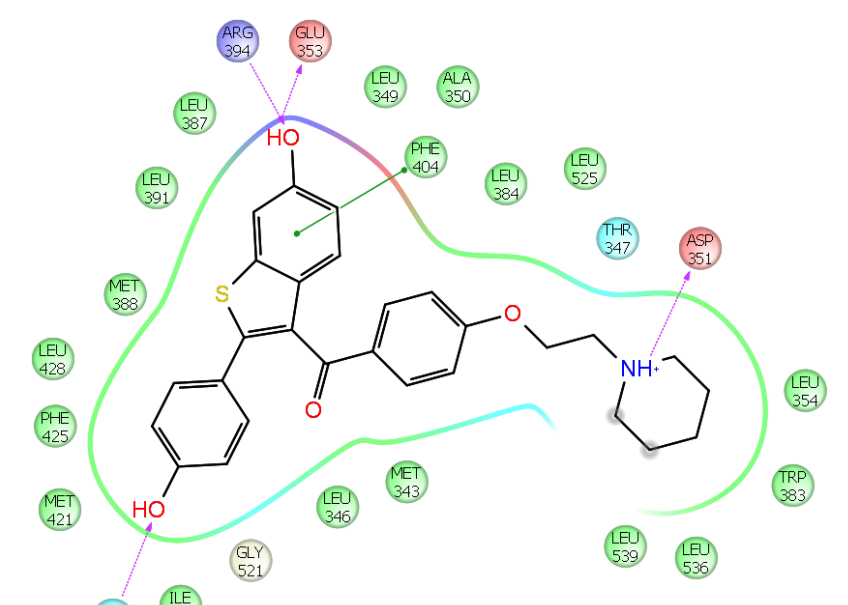

a) $\left({ }_{524}\left(\begin{array}{cc}1124 \\ 424\end{array}\right.\right.$

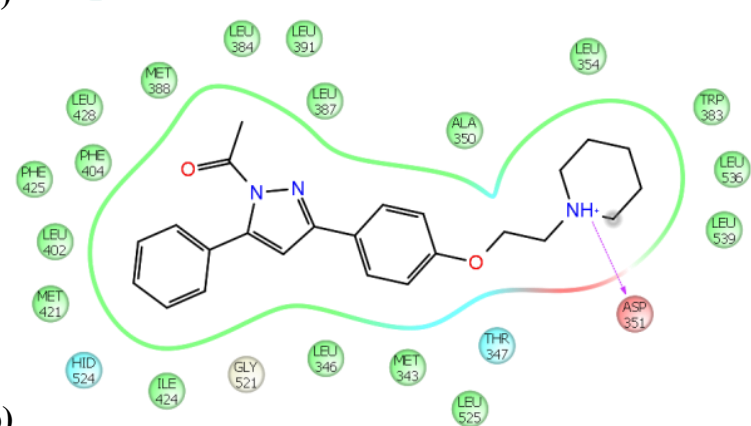

b)

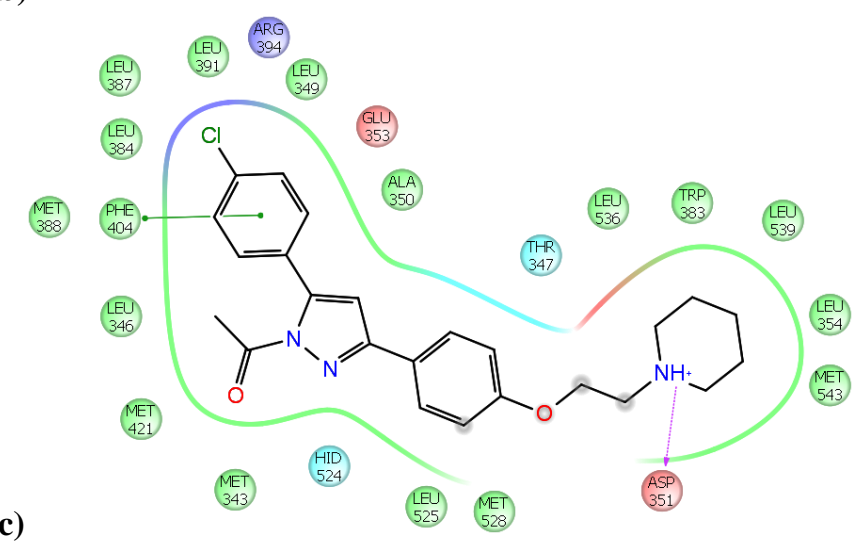

c)

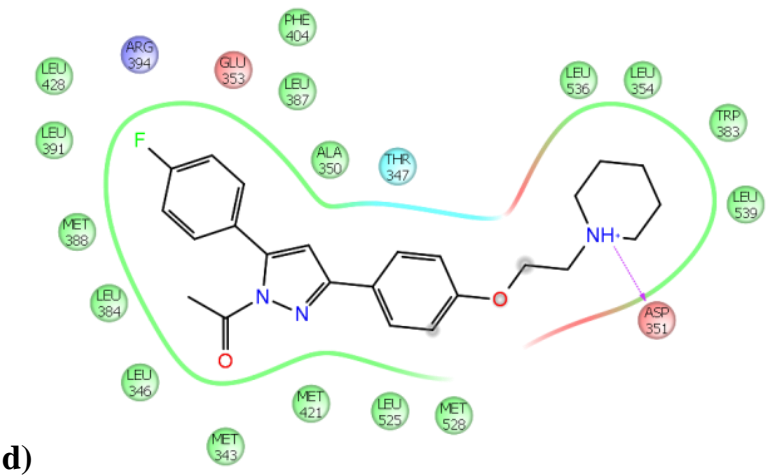

Figure 3: 2D Ligand Interaction diagram of (a) Raloxifen, (b) Compound 4a, (c) Compound $4 \mathrm{~b}$ and (d) Compound $4 g$.

b)
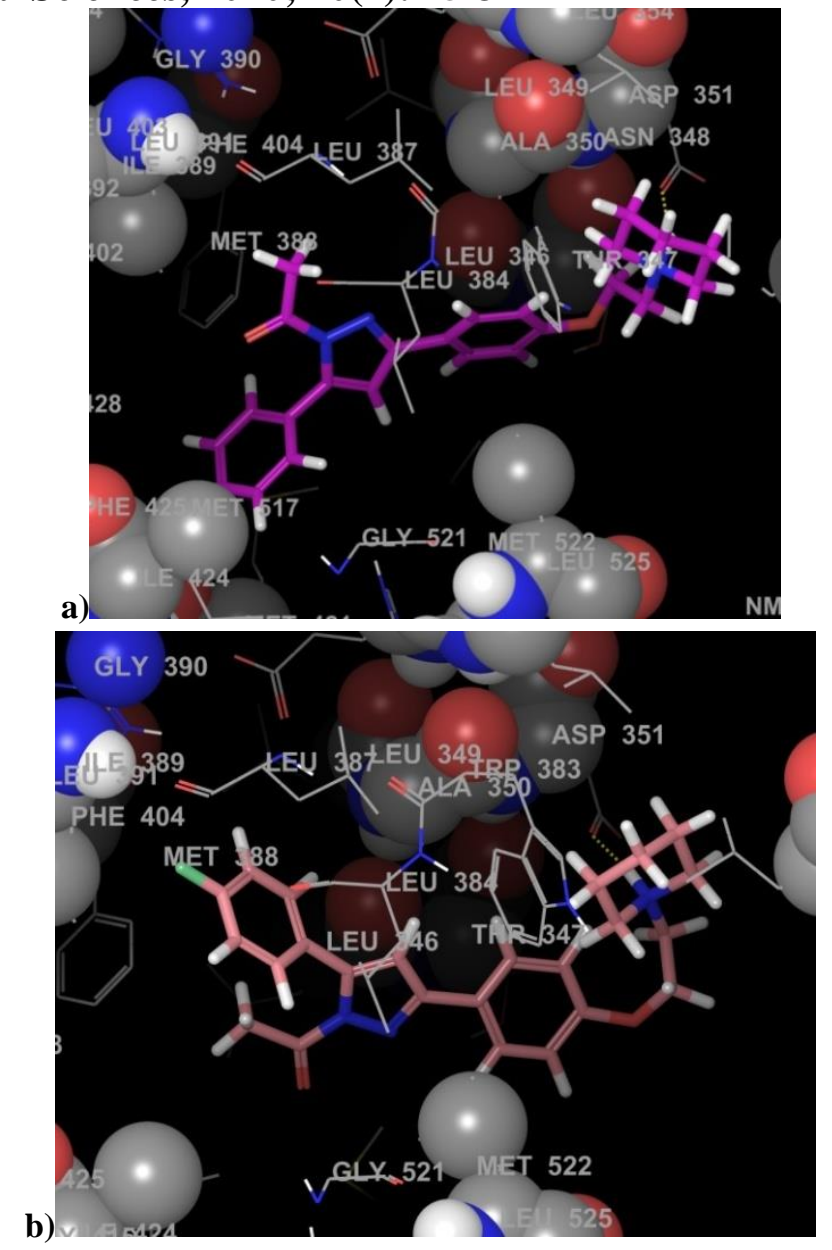

Figure 4: Docking analysis of (a) compound 4a and (b) compound $4 \mathrm{~g}$.

\section{CONCLUSION}

In summary a series of $\mathrm{N}$-aryl-2-pyrazolines derivatives were synthesized and evaluated for their binding activity with the estrogen receptor (ER) receptor and modulate it. The derivatives with electronegative substitution on the benzylidene motif turned out to be ineffective in the in vitro assays performed for evaluating the binding activity with the ER. The derivative with $p$-methoxy substitution on the benzylidene motif $(\mathbf{4 c})$ tends to bind effectively with the ER $\alpha$; its ability to bind with the receptor was also substantiated by in vivo activity.

\section{CONFLICT OF INTEREST}

The authors confirm that this article content has no conflict of interest.

\section{ACKNOWLEDGEMENT}

The authors are thankful to Tata Memorial Centre- Advanced Centre for Treatment, Research and Education in Cancer (ACTREC) Kharghar, Navi Mumbai for in vitro anti-breast 


\section{Indo Global Journal of Pharmaceutical Sciences, 2020; 10(1): 25-32}

cancer activity. The authors are also thankful to Y.B. Chavan College of Pharmacy, Aurangabad, Maharashtra for in vivo study. The authors are also thankful to SAIF, Panjab University, Chandigarh, India for providing the spectroscopy facility.

\section{REFERENCES}

1. American Cancer Society. Cancer Facts \& Figures 2019. Atlanta: American Cancer Society; 2019;3-4.

2. Liu, L., Tang, Z., Wu, C., Li, X., Huang, A., Lu, X., You, Q., Xiang, H. Synthesis and Biological evaluation of 4,6-diaryl-2-pyrimidinamine derivatives as anti-breast cancer agents. Bioorg. Med. Chem. Lett., 2018; 28:1138-1142. (doi.org/10.1016/j.bmcl.2017.12.066)

3. Bray, F., Ferlay, J., Soerjomataram, I., Siegel, R.L., Torre, L.A., Jemal, A. Global cancer statistics 2018: GLOBOCAN estimates of incidence and mortality worldwide for 36 cancers in 185 countries. CA Cancer J. Clin., 2018; 68:394-424. (doi: 10.3322/caac.21492)

4. Shagufta, Ahmad, I. Tamoxifen a pioneering drug: An update on the therapeutic potential of tamoxifen derivatives. Eur. J. Med. Chem., 2018; 143:515-531. (doi: 10.1016/j.ejmech.2017)

5. Gross, J.M., Yee, D. How does the estrogen receptor work? Breast Cancer Res., 2002; 4:62-64. (doi: 10.1186/bcr424)

6. Jia, M., Wright, K.D., Gustafsson, J.A. Estrogen receptor alpha and beta in health and disease. Clin. Endocrinol. Metab., 2015; 29:557-568. (doi: 10.1016/j.beem.2015.04.008)

7. Zhuang, C., Zhang, W., Sheng, C., Zhang, W., Xing, C., Miao, Z. Chalcone: A Privileged Structure in Medicinal Chemistry. Chem. Rev., 2017; 117:7762-810. (doi: 10.1021/acs.chemrev.7b00020)
8. Fustero, S., Rosello, M.S., Barrio, P., Fuentes, A.S. From 2000 to Mid2010: A Fruitful Decade for the Synthesis of Pyrazoles. Chem. Rev., 2011; 111:6984-7034. (doi.org/10.1021/cr2000459)

9. Ariazi, E.A., Clark, G.M., Mertz, J.E. Estrogen-related receptor alpha and estrogen-related receptor gamma associate with unfavorable and favorable biomarkers, respectively, in human breast cancer. Cancer Res., 2002; 62:6510-6518.

10. Mandal, S., Davie, J.R. An integrated analysis of genes and pathways exhibiting metabolic differences between estrogen receptor positive breast cancer cells. BMC Cancer, 2007; 7:181.

11. Dube, P.N., Mokale, S.N. Design and Synthesis of Some Novel Estrogen Receptor Modulators as Anti-Breast Cancer Agents: In Vitro \& In Vivo Screening, Docking Analysis. Anti-cancer Agents Med. chem., 2017; 16(11):1461-1467. (DOI: 10.2174/1871520616666160211124617)

12. Skehan, P., Storeng, R., Scudiero, D., Monks, A., McMohan, J., Vistica, D., Jonathan, T.W., Warren, J.T., Bokesch, H., Kenny, S., Boyd, M.R. New colorimetric cytotoxicity assay for anticancer-drug screening. J. Natl. Cancer Inst., 1990; 82:1107-12.

13. Teplitzky, S.R., Kiefer, T.L., Cheng, Q., Dwivedi, P.D., Moroz, K., Myers, L., Anderson, M.B., Collins, A., Dai, J., Yuan, L., Spriggs, L.L., Blask, D.E., Hill, S.M. Chemoprevention of NMU-induced rat mammary carcinoma with the combination of melatonin and 9-cis-retinoic acid. Cancer Lett., 2001; 168:155-163. (doi.org/10.1016/S03043835(01)00548-1)

14. Schrödinger Release 2019-1: Glide, Schrödinger, LLC, New York, NY, 2019. 